\title{
OCORRÊNCIA DE DINOFLAGELADOS TECADOS POTENCIALMENTE TÓXICOS E NOCIVOS EM CULTIVOS DE MOLUSCOS SITUADOS NO MUNICÍPIO DE PENHA, SC
}

\author{
MIOTTO, M. C. ${ }^{1} \&$ TAMANAHA, M. S. ${ }^{2}$ \\ Laboratório de Algas Nocivas, Centro de Ciências Tecnológicas da Terra e do Mar - CTTMar, \\ Universidade do Vale do Itajaí - UNIVALI, Itajaí, SC, Brasil \\ 1 - cecilia.miotto@gmail.com; 2 - mstamanaha@gmail.com
}

\begin{abstract}
Miotto, M. C. \& Tamanaha, M. S. (2012) Occurrence of potentially toxic and harmful thecate dynoflagellates in mussel farms at Penha municipality, SC. Braz. J. Aquat. Sci. Technol. 16(1): 53-67. elSSN 1983-9057. The purpose of this study was to describe the composition and occurrence of potentially toxic and harmful species of thecate dinoflagellates in cultured shellfish, located at Armação do Itapocorói (AI) and Praia Alegre (PA) in the municipality of Penha, SC - Brazil. Water samples were collected and environmental parameters (salinity, water temperature and depth of Secchi disk) were determined weekly between April 2007 and April 2008 at two sites by a monitoring program maintained by the Laboratory for the Study of Harmful Algae, CTTMar, UNIVALI. The identification of the organisms was made through an Olympus microscope, model BX41 with up to 1000x. During the sampling period, we identified 21 species of thecate dinoflagellates potentially toxic and harmful in the genus Alexandrium, Ceratium, Dinophysis and Prorocentrum. The species $P$. obtusum $(86 \%), D$. acuminata $(78.5 \%)$ and $P$. micans $(72 \%)$ were very frequent throughout the period in the two sample points. The point with highest species richness was the Al, with 14 species, and the PA had the lowest richness with only two species. Most samples were considered rare relative to the abundance of thecate dinoflagellates compared to the presence of harmful microalgae, but during the month of September 2007, were considered abundant, during which there was a bloom of $D$. acuminata. The similarity between the two points throughout the sampling period reached a high coefficient $(86 \%)$, and between the months examined, June 2007 showed the lowest similarity (10\%) and July 2007 the greatest similarity recorded (91\%). The composition and distribution of thecate dinoflagellates during the study showed that the existence of a dynamic community structure could be used as a tool to aid the monitoring of harmful algae mariculture activity in this region.
\end{abstract}

Keywords: harmful algae, dinoflagellates, mariculture.

\section{INTRODUÇÃO}

Devido às suas condições oceanográficas propícias ao desenvolvimento do mexilhão, a mitilicultura no Estado de Santa Catarina se difundiu em praticamente todas as enseadas e baías da costa centro-norte e a região se tornou, na última década, o maior produtor de mexilhões da América Latina (Marenzi \& Branco, 2006). A primeira referência de cultivo de mexilhões Perna perna Linnaeus 1758 para o Estado de Santa Catarina foi em 1985 na Enseada da Armação do Itapocoroy no município de Penha, onde, atualmente, está localizado um dos maiores parques de cultivo de moluscos(Panorama da Aquicultura, 2001), representando $17,65 \%$ da produção de mexilhões cultivados no estado (Oliveira Neto, 2007).

Em decorrência deste rápido crescimento, tornou-se necessário garantir a qualidade e a segurança do produto, principalmente devido aos eventos de florações de algas nocivas registrados no estado. Somente no episódio de floração em 2007, mais de 150 casos de intoxicação por frutos do mar contaminados foram registrados em Santa Catarina (Proença et al., 2007). Desta forma, este trabalho teve como objetivo enriquecer o conhecimento das espécies de dinoflagelados tecados no litoral centro-norte de Santa Catarina, a fim de registrar informações da existência de muitas espécies com risco potencial de formação de florações na zona costeira, sobretudo em áreas de cultivo de moluscos marinhos.

\section{MATERIAL E MÉTODOS}

\section{Área de Estudo}

A enseada de Armação do Itapocorói (AI) $\left(26^{\circ}\right.$ $\left.36^{\prime} 08^{\prime \prime} S, 48^{\circ} 36^{\prime} 29^{\prime \prime} \mathrm{W}\right)$ e a Praia Alegre (PA) $\left(26^{\circ} 45^{\prime}\right.$ $46^{\prime \prime}$ S, $48^{\circ} 39^{\prime} 07^{\prime \prime} \mathrm{W}$ ) estão localizadas no município de Penha, estado de Santa Catarina, região sul do Brasil e distantes aproximadamente $5 \mathrm{~km}$ entre si (Figura 1). A salinidade na Enseada de Armação do Itapocorói é em torno de 34 e temperaturas entre $19^{\circ}$ e $28^{\circ} \mathrm{C}$. (Schettini et al., 1999). O ponto amostral situado no cultivo de moluscos em PA é regido por condições abióticas semelhantes ao de Al, contudo, está sob forte influência de dois aportes fluviais.

\section{Amostragem}

As amostras foram coletadas semanalmente na baía da Armação do Itapocorói e Praia Alegre 


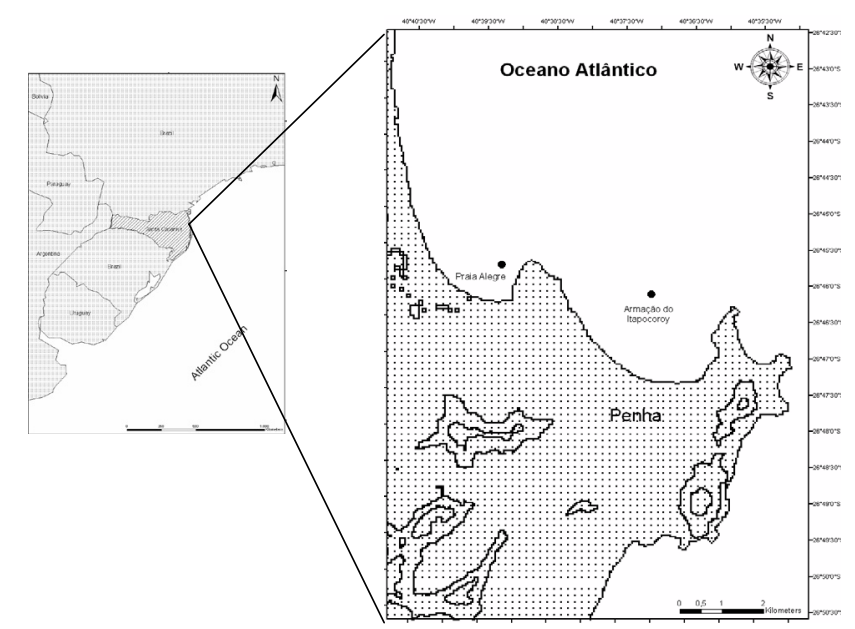

Figura 1 - Localização das áreas de cultivo de moluscos onde foram coletadas as amostras, $(\bullet)$ Enseada de Armação do Itapocorói, e $(\bullet)$ Praia Alegre, Penha, SC.

através do monitoramento mantido pelo Laboratório de Estudos sobre Algas Nocivas, CTTMar, UNIVALI, durante o período de abril de 2007 à abril de 2008.

A salinidade e a temperatura da água foram obtidas através de um salinômetro com termômetro digital marca YSI Yellow Springs modelo 30/10 FT. A transparência da água foi mensurada através do desaparecimento do Disco de Secchi branco com 20 $\mathrm{cm}$ de diâmetro.

As amostras foram coletadas com rede de plâncton (malha de $20 \mu \mathrm{m}$ ), sendo $100 \mathrm{ml}$ fixados com Formaldeído $4 \%$ para posterior análise em microscópio óptico.

\section{Análise do Material Biológico}

As espécies foram identificadas em microscópio óptico trinocular com contraste de fase marca Olympus, modelo BX41 com aumento de até 1000x, onde também foram tomadas fotomicrografias com o auxílio de uma câmera digital Sony 7.2 megapixels. As seguintes bibliografias foram utilizadas para o auxílio na identificação dos dinoflagelados: Balech et al. (1984); Balech (1988); Fukuyo et al. (1990); Larsen \& Moestrup (1992); Balech (1995); Steidinger \& Tangen (1997); Cardoso (1998); Faust et al. (1999); Faust \& Gulledge (2002); Taylor et al. (2003); Larsen \& Ngueyen (2004).

As variáveis biológicas foram plotadas e processadas em planilha Excel $\circledast$ e posteriormente calculadas: (a) a riqueza de espécies; (b) a freqüência de ocorrência, calculada de acordo com Lobo \& Leighton (1986) onde: $>70 \%$, considerada muito freqüente; $\leq 70 \%$ a $>30 \%$, freqüente; $\leq 30 \%$ a $>10 \%$, pouco freqüente; $\leq 10 \%$, esporádica; (c) a abundância relativa (\%), a qual representa a abundância de uma espécie comparada com o fitoplâncton total, classificando as espécies nas seguintes categorias (Omori \& Ikeda,
1984): $>70 \%$ dominante; $\leq 70 \%$ a $>40 \%$ abundante; $\leq 40 \%$ a $>10 \%$ pouco abundante e $\leq 10 \%$ rara; e (d) o Índice de similaridade de Jaccard (Sj) que mede a semelhança entre dois pontos baseado na comparação de suas espécies onde, $\mathrm{Sj}=0 \%$ - nenhuma similaridade e $\mathrm{Sj}=100 \%$ - total similaridade.

\section{RESULTADOS}

\section{Temperatura, Salinidade e Transparência da} água.

A salinidade no ponto $\mathrm{Al}$ apresentou uma média de $30 \pm 2,8$, tendo um valor mínimo de 25,4 em 25/07/2007 e um valor máximo de 36 em 17/01/2008. O ponto PA apresentou uma média de $29,7 \pm 2,6$, tendo um valor mínimo de 24,5 em 25/07/2007 e um valor máximo de 36 em 17/01/2008. Durante os meses de julho a outubro de 2007 foi registrada uma queda acentuada nos valores de salinidade em ambos os pontos (Figura 2).

A temperatura da água apresentou média de $20,5^{\circ} \mathrm{C} \pm 3,7^{\circ} \mathrm{C}$, tendo um valor mínimo de $15,6^{\circ} \mathrm{C}$ em 27 e 30/07/2007 e um valor máximo de $27^{\circ} \mathrm{C}$ em $17 / 01 / 2008$ no ponto Al e no ponto PA a média foi de $20,8 \pm 3,8^{\circ} \mathrm{C}$, tendo um valor mínimo de $15,4^{\circ} \mathrm{C}$ em 25/07/2007 e um valor máximo de $27^{\circ} \mathrm{C}$ em 6 e 19/03/2008 (Figura 3).

A transparência da água no ponto $\mathrm{Al}$ apresentou uma média de 2,9 91,2 metros, tendo um valor máximo de 7 metros em 31/10/2007 e um valor mínimo de 1,0 metro em 17/05/2007. No ponto PA, a transparência da água apresentou uma média de 1,7 $\pm 0,7$ metros, tendo um valor máximo de 3,4 metros em 05/07/2007 e um valor mínimo de 0,3 metro em 19/04/2007 (Figura 4).

\section{Variáveis biológicas}

Durante o período estudado, foram identificadas 20 espécies de dinoflagelados tecados potencialmente tóxicos e/ou nocivos em Armação do Itapocorói (AI) e Praia Alegre (PA), sendo dez espécies pertencentes ao gênero Prorocentrum (Figuras 5-6), oito ao gênero Dinophysis (Figuras 7-8), uma ao gênero Alexandrium (Figura 9a-b) e uma ao gênero Neoceratium (Figura 9c), listadas na Tabela 1.

A riqueza específica teve uma média de 7,5 espécies em Al, com um máximo de 13 e um mínimo de três espécies (Figura 10), apresentando a maior riqueza específica registrada em todo o período amostral. No ponto PA, foi observada uma média de 6,3 espécies, com máxima de 11 e mínima de uma espécie (Figura 10), sendo a menor riqueza específica registrada.

Entre os gêneros, o mais representativo durante todo o período amostrado em relação à riqueza 
Braz. J. Aquat. Sci. Technol., 2012, 16(1):53-67.



Figura 2 - Variação da salinidade entre os meses de abril de 2007 e abril de 2008 nos pontos PA e Al.

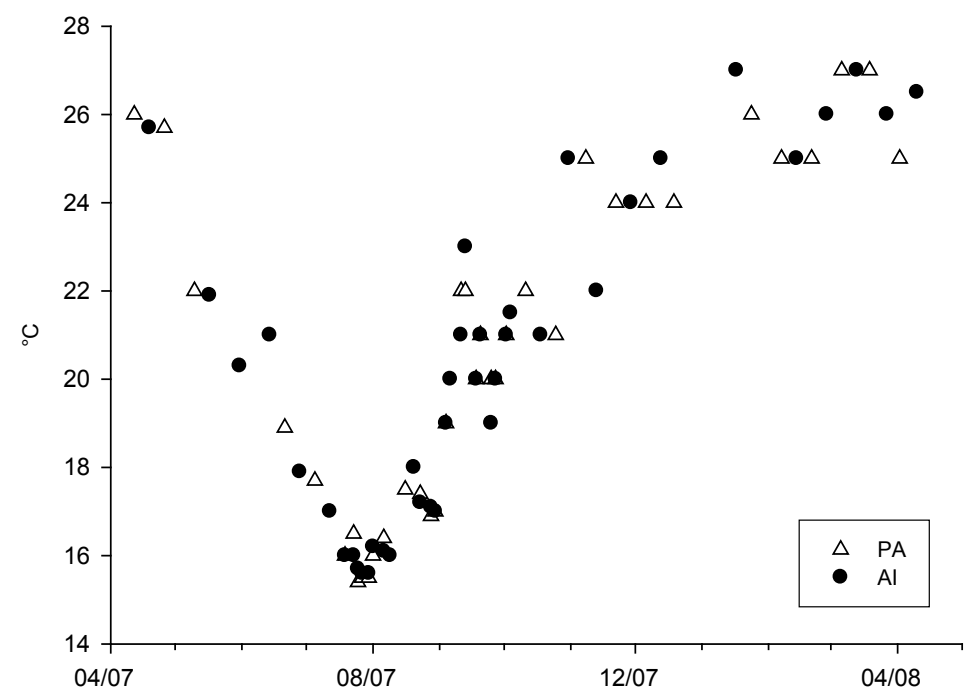

Figura 3 - Variação da temperatura da água entre os meses de abril de 2007 e abril de 2008 nos pontos PA e Al.

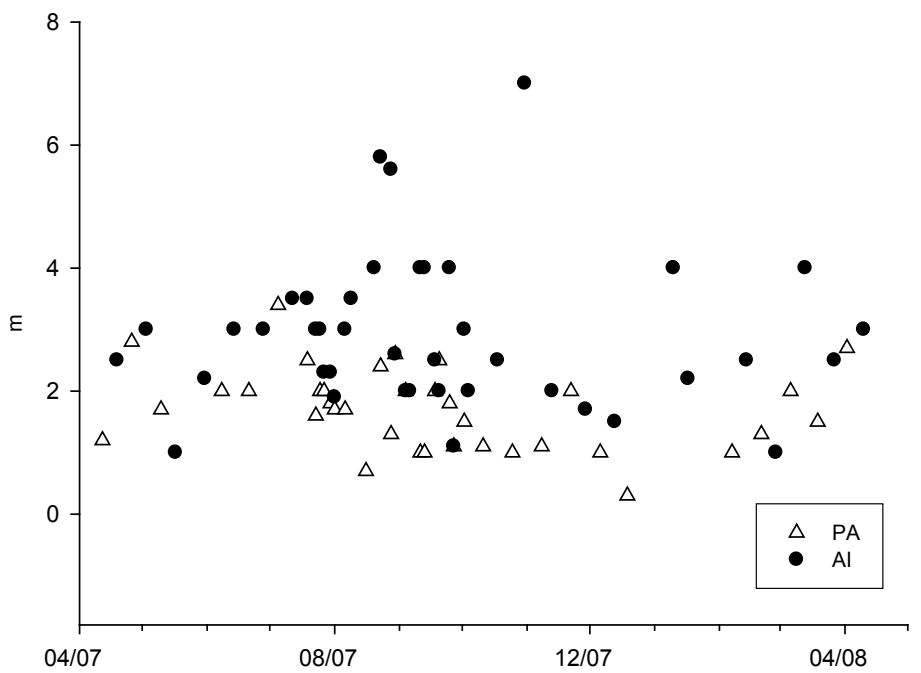

Figura 4 - Variação da medida do Disco de Secchi entre os meses de abril de 2007 e abril de 2008 nos pontos PA e Al. 

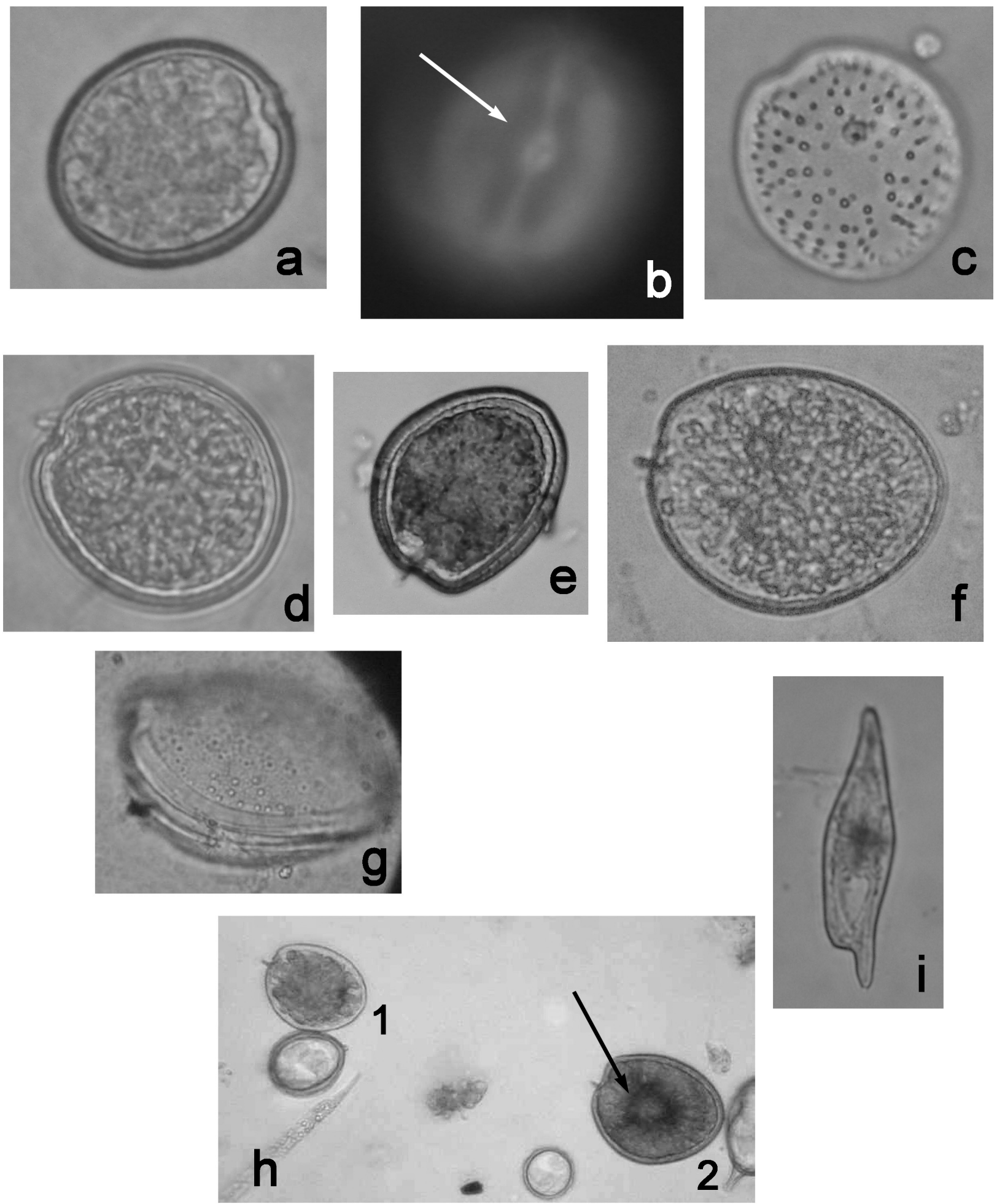

Figura 5 - Gênero Prorocentrum. (a-b) Prorocentrum compressum, a: vista valvar; b: região periflagelar, em detalhe poro apical; (c-d) $P$. obtusum, c: vista valvar em detalhe a estrutura dos poros; d: vista valvar; (e) P. mexicanum; (f-g) P. rhathymum, f: vista valvar; g: estrutura dos poros; (h) 1. P. rhathymum e 2. P. mexicanum, em detalhe (seta) presença do pirenóide central; (i) P. rostratum, vista valvar. Figs. a, c, d, e, f, g, h, i: M. O.; Fig. b: M. Epifl. (g) aumento 1000X; (a, b, c, d, e, f, i) aumento 400X; (h) aumento 200X. 

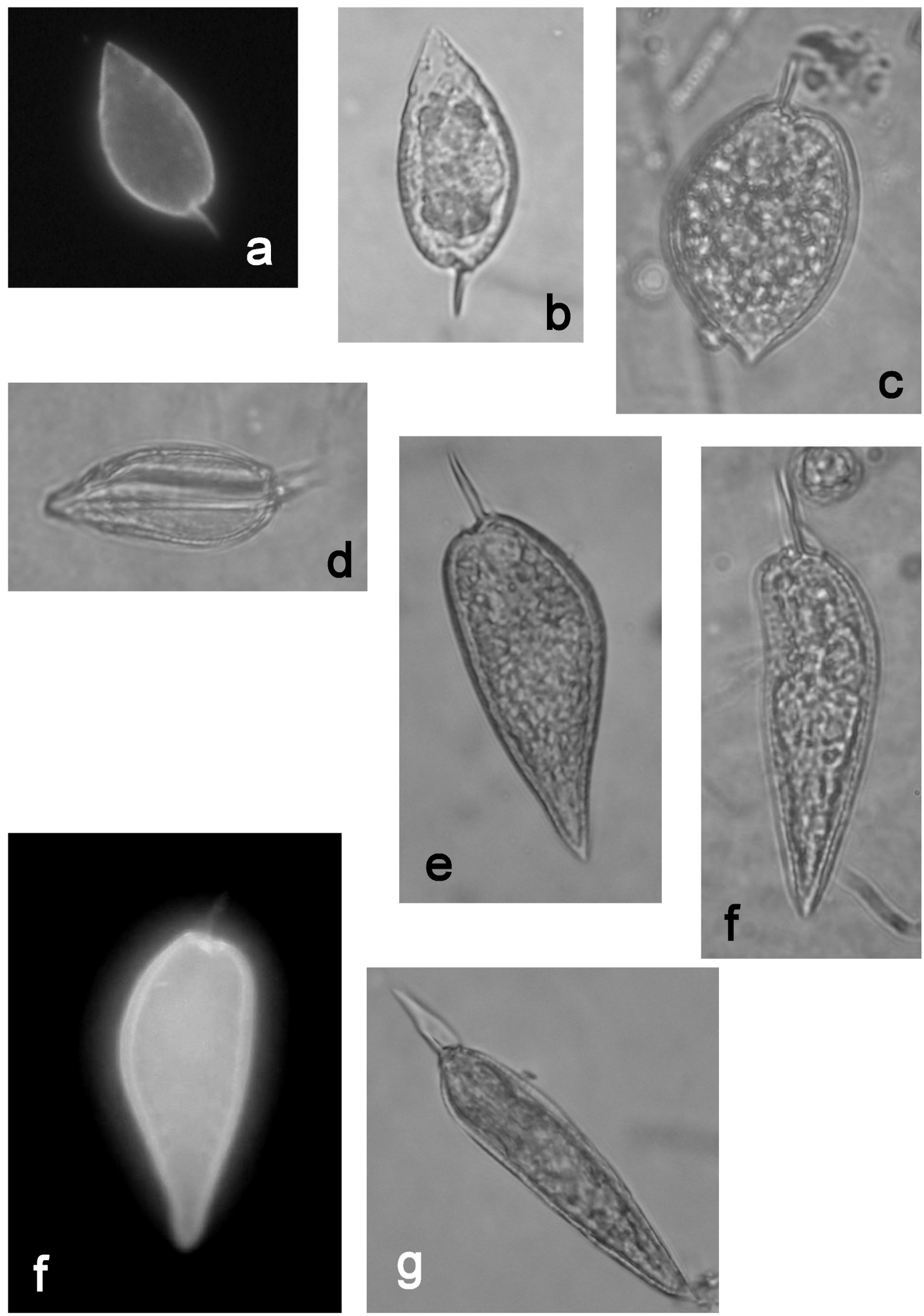

Figura 6 - Gênero Prorocentrum. (a-d) P. micans, a: vista valvar mostrando a estrutura dos poros; b-c: variações morfológicas; d: vista lateral; (e-g) P. gracile, e: vista valvar; f: vista valvar mostrando a estrutura de poros; g; vista lateral; (h) $P$. sigmoides, vista valvar. Figs. b, c, d, e, g, h: M. O.; Figs. a, f: M. Epifl. Aumento de 400X. 

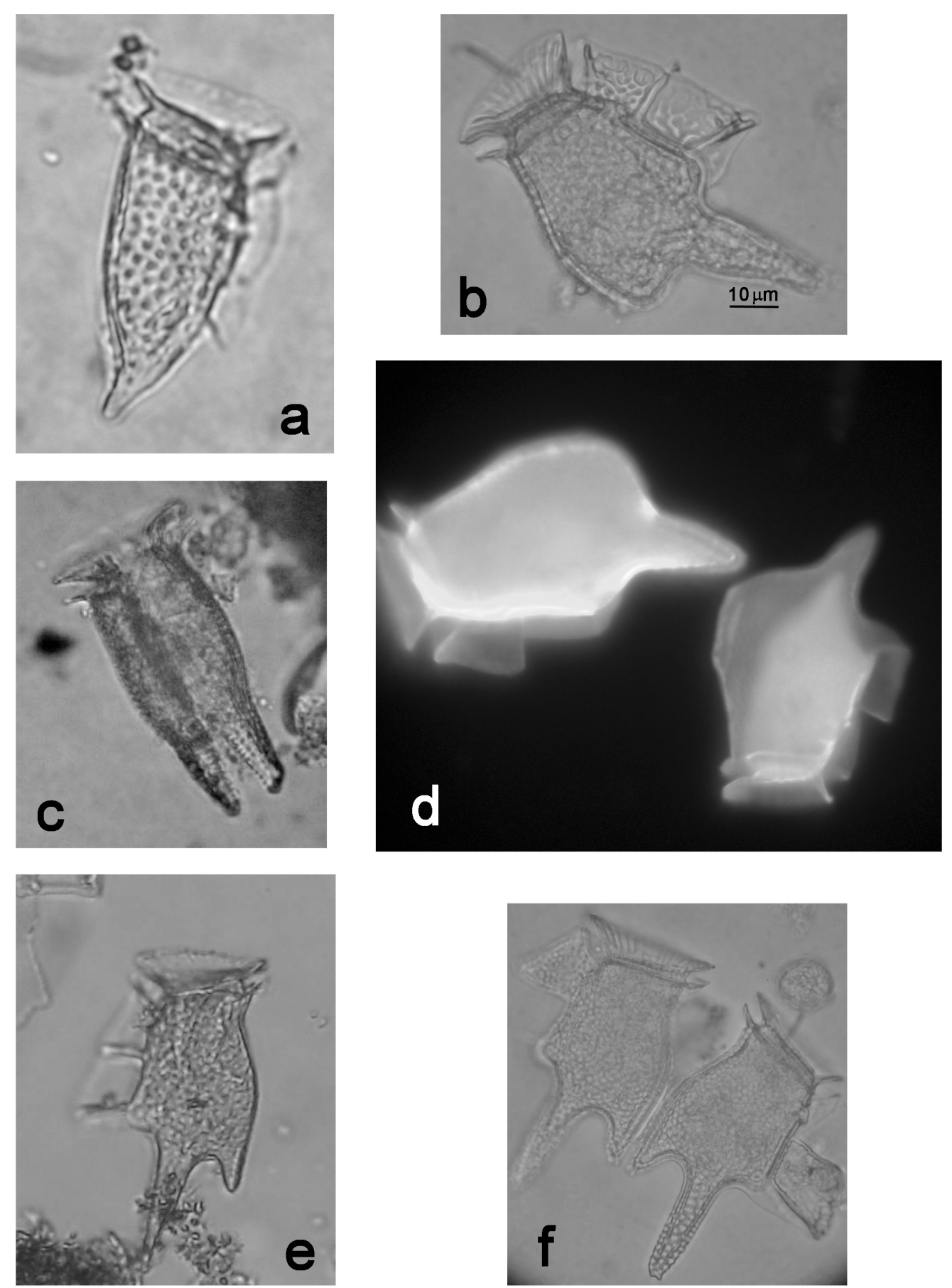

Figura 7 - Gênero Dinophysis. (a) D. diegensis; (b-d) D. caudata, b: vista lateral; c: células em divisão; d: vista lateral; (e-f). D. tripos, e: vista lateral; f: células em divisão; Figs. a, b, c, f, e: M.O.; Fig. d: M. Epifl. (a, c, e) aumento de 200X, (b, d, f) aumento de 400X.

específica no ponto PA foi Prorocentrum (60\%), o segundo foi Dinophysis (33\%) e o terceiro Neoceratium (6\%) (Figura 11). No ponto Al, o gênero mais representativo também foi Prorocentrum (55\%), seguido de Dinophysis (34\%) e o terceiro Neoceratium (10\%) (Figura 11).

Durante o período amostral, ocorreu um evento de floração de $D$. acuminata, o qual propiciou uma queda na riqueza de espécies, na metade do mês de julho. Neste período, o gênero Dinophysis foi o mais representativo na riqueza específica, mas os gêneros Neoceratium e Prorocentrum continuaram sempre presentes, sendo algumas vezes Prorocentrum mais representativo que Dinophysis.

Os gêneros Prorocentrum e Dinophysis foram considerados muito freqüentes em todo o 

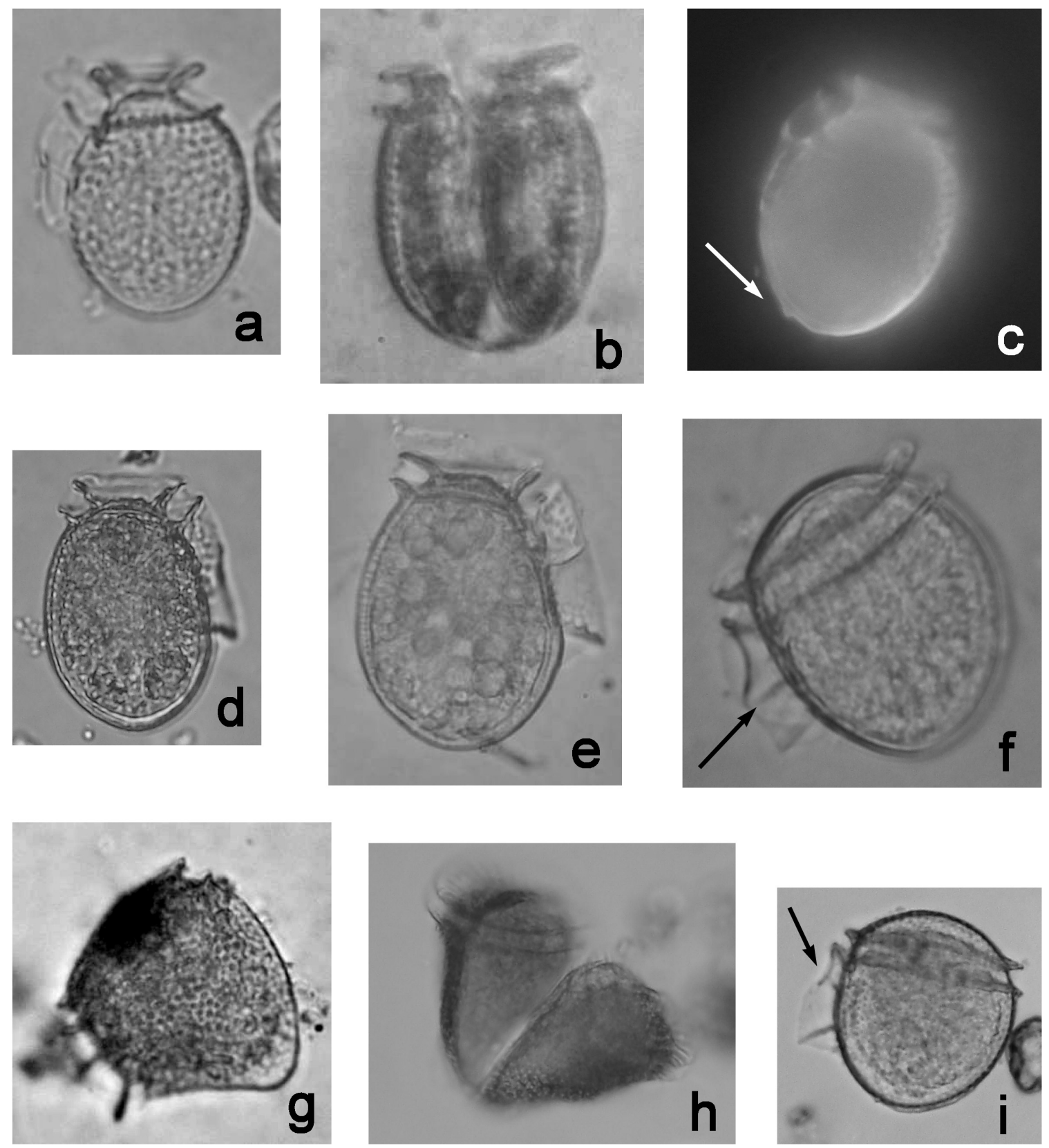

Figura 8 - Gênero Dinophysis. (a-c) D. acuminata, a: vista lateral; b: células em divisão; c: variações morfológicas, presença de uma verruga (seta); (d-e) D. fortii, vista lateral; (f) D. scrobiculata, posição da R2 mais próxima a R3; (g-h) D. mitra, g: vista lateral; h: células em divisão; (i) D. rotundata, posição da R2 mais próxima a R1. Figs. a, b, d, e, f, g, h, i: M.O.; Fig. c: M. Epifl. (a, b, c, e, f, g, h, i) aumento de 400X. (d) aumento 200X.

período amostrado e as espécies que apresentaram as maiores freqüências de ocorrência foram Prorocentrum obtusum (89,9\%), Dinophysis acuminata $(79,7 \%)$ e Prorocentrum micans $(77,2 \%)$ sendo muito freqüentes nos dois pontos amostrados (Figura 12).

O ponto que apresentou a maior abundância relativa foi o ponto Al em 25/09/07 onde a porcentagem de dinoflagelados tecados nocivos foi $64,9 \%$ comparada à presença de outras microalgas. No ponto PA, a maior abundância relativa foi registrada em 12/04/07 com 61,6\% (Figura 13).

Na maior parte das amostras $(45,9 \%)$ foi considerada rara a abundância relativa dos dinoflagelados tecados nocivos comparados à presença de outras microalgas. Esta relação foi influenciada provavelmente pela dominância de diatomáceas, que estiveram presentes em algumas amostras de julho de 2007 e mais freqüentes nas amostras a partir do final de 

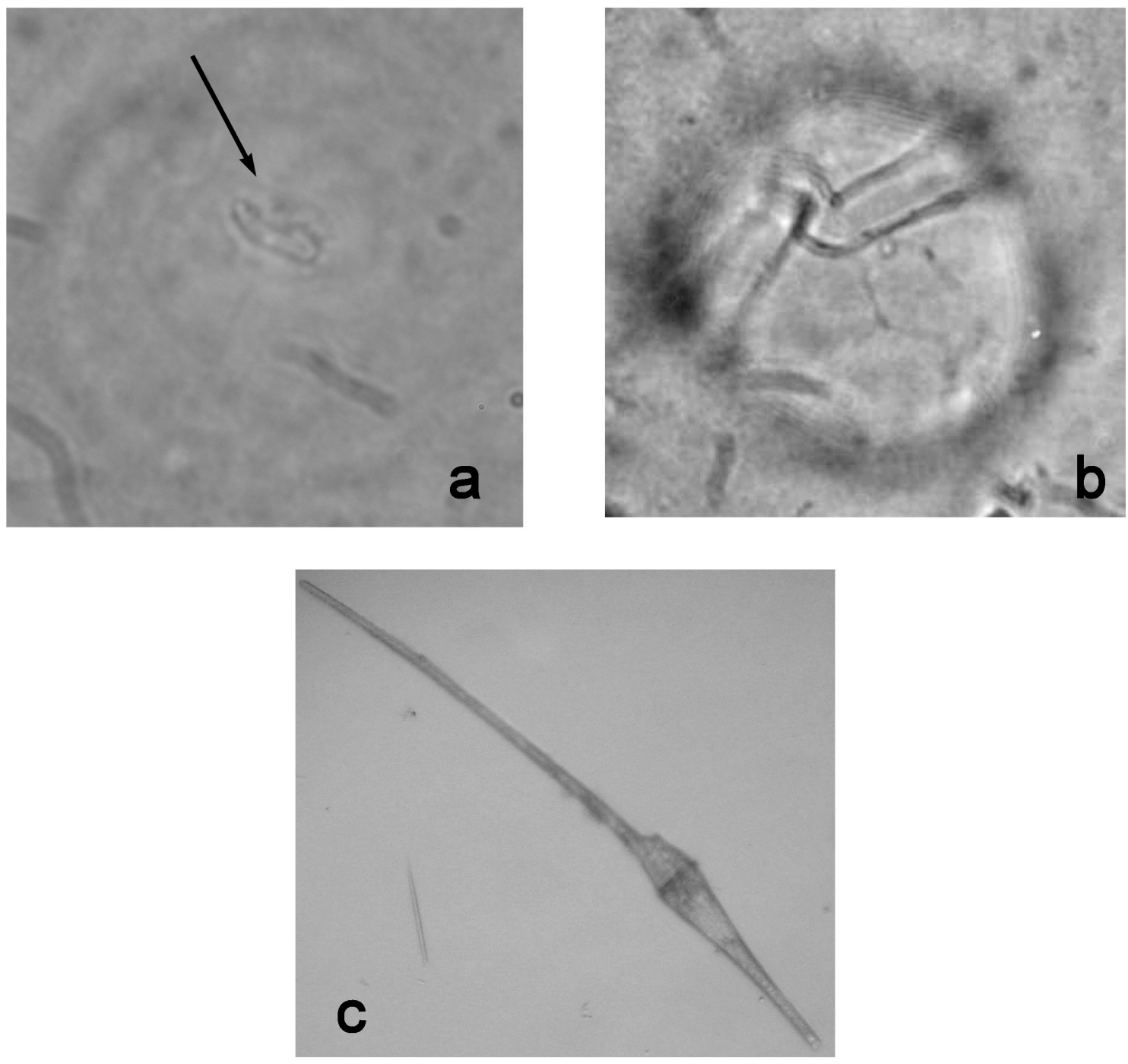

Figura 9 - Gênero Alexandrium. (a-b) A. cf. tamarense, a: em detalhe (seta) o APC; b: vista geral da célula; (c). Gênero Neoceratium. (c) N. fusus, vista geral. Figs. a, b, c: M. O.; (a, b) aumento 1000X; (c) aumento 400X.

outubro de 2007 até o início de abril de 2008, período típico de florações de diatomáceas na área de estudo.

A similaridade entre os dois pontos obteve um coeficiente alto, $86 \%$, segundo o índice de similaridade de Jaccard (Sj). O mês de junho de 2007 apresentou a menor similaridade (10\%), e o mês de julho de 2007 , a maior similaridade $(91 \%)$ entre os meses observados.

\section{DISCUSSÃO}

A variação da temperatura registrada durante o período amostral obedece a uma distribuição típica da região com predomínio de clima subtropical, como verificado em outros trabalhos (Rörig et al., 1998; Schettini et al., 1999; Schimitt \& Proença, 1999). A salinidade média registrada condiz com Schettini et al. (1999) que descreveram que a salinidade na área de estudo é raramente maior que 34 e é influenciada principalmente por aporte continental e pelos três tipos de massas de água que ocorrem no litoral centronorte de Santa Catarina, sendo a Água Costeira com salinidades baixas $(<34)$ que predomina no local. Os valores de transparência da água (medida do disco de Secchi) tiveram uma média de 2,3 metros, sendo que o ponto PA, por sofrer com o aporte continental diretamente apresentou valores menores que o ponto Al, isto pode estar relacionado também com a diferença na profundidade dos locais de amostragem, sendo que PA tem no máximo $6 \mathrm{~m}$ de profundidade e o ponto de coleta de Al, 12 metros. Ou seja, a hidrodinâmica em interação com mais freqüente com o fundo em PA pode gerar ressuspensão, o que afetaria em parte a transparência da água. 
Tabela 1 - Lista de espécies e sua ocorrência no parque de maricultura em Al e PA, Penha, SC.

\begin{tabular}{|c|c|c|}
\hline Ordem & Gênero & Espécies \\
\hline Gonyaulacales Taylor 1980 & Alexandrium Halim 1960 & A. cf. tamarense (Lebour) Balech 1971 \\
\hline Peridiniales Haeckel 1894 & $\begin{array}{l}\text { Neoceratium (Ehrenberg) } \\
\text { F.Gomez, D.Moreira \& } \\
\text { P.Lopez-Garcia } 2009\end{array}$ & $\begin{array}{l}\text { N. fusus (Ehrenberg) } \\
\text { F.Gomez, D.Moreira \& } \\
\text { P.Lopez-Garcia } 2009\end{array}$ \\
\hline $\begin{array}{l}\text { Dinophysiales Lindermann } \\
1928\end{array}$ & Dinophysis Ehrenberg 1938 & $\begin{array}{l}\text { D. acuminata Claparède et Lachmann } 1859 \\
\text { D. caudata Saville-Kent } 1881 \\
\text { D. fortii Pavillard } 1923 \\
\text { D. diegensis Kofoid } 1907 \\
\text { D. mitra Balech } 1967 \\
\text { D. rotundata Claparède et Lachmann } 1859 \\
\text { D. scrobiculata Balech } 1971 \\
\text { D. tripos Gourret } 1883\end{array}$ \\
\hline Prorocentrales Taylor 1980 & Prorocentrum Ehrenberg 1834 & $\begin{array}{l}\text { P. gracile Schutt } 1895 \\
\text { P. micans Ehrenberg } 1834 \\
P . \text { mexicanum Tafall } 1942 \\
\text { P. obtusum Ostenfeld } 1908 \\
P . \text { rhathymum Loeblich } 1979 \\
\text { P. compressum Abe ex Dodge } 1975 \\
\text { P. minimun (Pavillard) Schiller } 1933 \\
P . \text { rostratum Stein } 1883 \\
P . \text { sigmóides Bohm } 1933 \\
P \text {. dentatum Stein } 1883\end{array}$ \\
\hline
\end{tabular}

Pode-se observar que a riqueza de espécies e a abundância relativa dos dinoflagelados tecados tiveram uma relação inversa com a salinidade. À medida que a salinidade diminuiu, a riqueza de espécies aumentou, assim como no período de maior abundância relativa dos dinoflagelados tecados, a salinidade apresentou os menores valores.

Os gêneros Prorocentrum e Dinophysis que foram considerados muito freqüentes, são também destacados por Rörig et al. (1998) como os gêneros que apresentam espécies mais comuns na área de estudo, sendo as espécies $P$. obtusum e $P$. micans as mais freqüentemente registradas. Segundo Proença et al. (1999), essas espécies não são produtoras de toxinas DSP (ex.: ácido okadáico), porém devido a grande freqüência que estão ocorrendo além da alta abundância relativa, é de grande importância o registro destas espécies nos cultivos de moluscos da região.

Diversas espécies dos gêneros Prorocentrum e Dinophysis são produtoras de DSP e representam um risco potencial para contaminação da carne de moluscos. Segundo trabalhos de Lopez-Rodas (2006) e Manerio et al. (2008), as toxinas DSP parecem se comportar como agentes cancerígenos, principalmente em casos de câncer de colo retal.

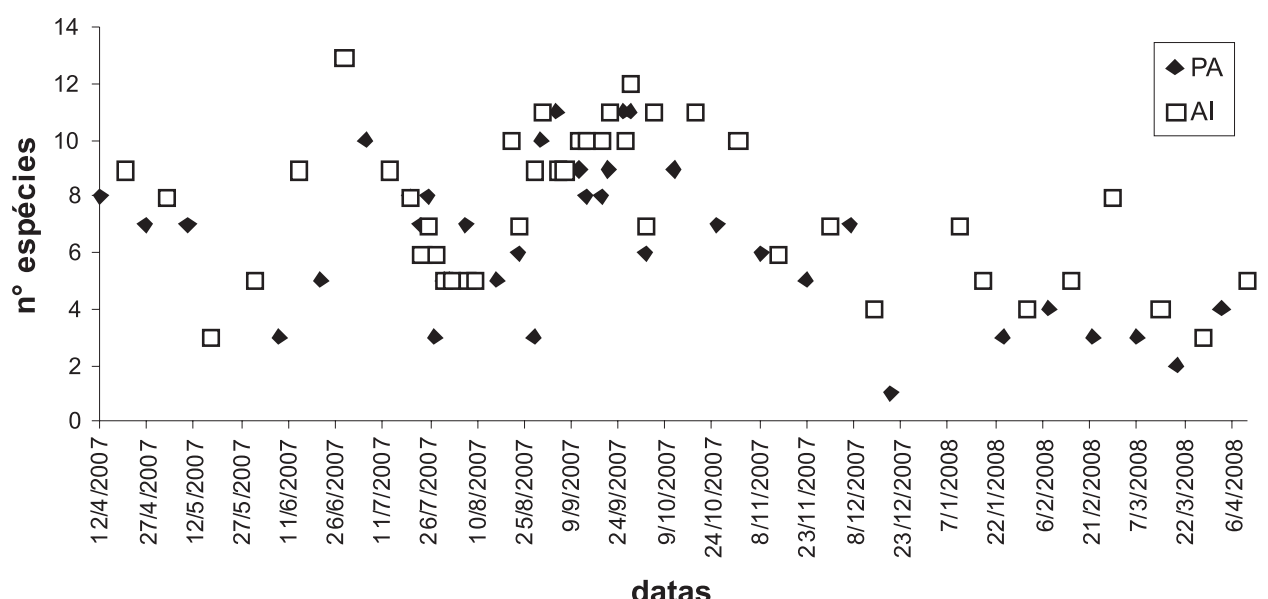

Figura 10 - Variação da riqueza específica de dinoflagelados tecados potencialmente tóxicos e nocivos durante o período de abril de 2007 e abril de 2008, nos pontos Al e PA. 
PA

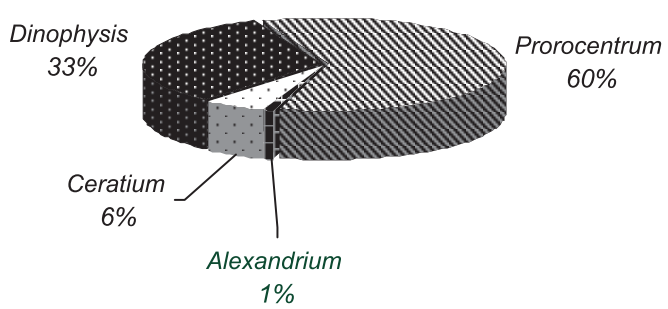

Al



Figura 11 - Percentual de riqueza específica dos gêneros de dinoflagelados tecados potencialmente tóxicos e nocivos no ponto PA e Al.

\section{Espécies muito freqüentes}

$D$. acuminata é uma espécie tóxica associada com eventos de DSP, descrita como sendo de ampla distribuição mundial, e aparentemente mais abundante em águas costeiras, especialmente as eutrofizadas (Taylor et al., 2003). As células observadas apresentaram 22-35 $\mu \mathrm{m}$ de comprimento e 17,5-32,5 $\mu \mathrm{m}$ de largura sendo menores que as descritas na literatura, e características morfológicas variadas como diferentes tamanhos e a presença de uma até três verrugas na hipoteca. D. acuminata esteve presente em $79,4 \%$ das amostras, porém, durante sua ausência espécies do gênero Prorocentrum estiveram sempre presentes destacando-se a espécie $P$. obtusum.

Eventos de florações tóxicas de $D$. acuminata já foram descritos em vários países da América do Sul, no Brasil, sua presença foi registrada no Rio de Janeiro (Tenenbaum et al., 2004), Paraná (Mafra Jr. et al., 2006) Santa Catarina (Proença et al., 1998, 1999, 2007) e no Rio Grande do Sul (Odebrecht et al., 2002).

P. obtusum, segundo Guimarães \& Rorig (2004) é uma das duas espécies de Prorocentrum que mais ocorrem na costa de Santa Catarina. As células encontradas no presente estudo são similares ao descrito por Balech (1984) proveniente de Atlântico Sul Ocidental, apresentando 35-40 $\mu \mathrm{m}$ de comprimento e 25-35 $\mu \mathrm{m}$ de largura. P. obtusum apresentou $89,9 \%$ de freqüência de ocorrência, a maior registrada no presente trabalho. Sua toxicidade ainda é desconhecida segundo Harsen et al. (2001), porém análises de toxinas diarréicas realizadas por Proença et al. (1999), na Armação do Itapocorói, indicam que esta espécie não é produtora de toxina da DSP.

P. micans ocorreu em $77,2 \%$ das amostras. As células encontradas apresentaram 40-60 $\mu \mathrm{m}$ de comprimento 20-25 $\mu \mathrm{m}$ de largura, com diferentes variações morfológicas. É uma das mais comuns e diversificadas espécies do gênero Prorocentrum, comumente encontrada em águas neríticas e estuarinas, mas também em ambientes oceânicos (Faust \& Gulledge, 2002). No Brasil, foi descrita por Cardoso (1998) em Santa Catarina, Rörig et al. (1998) e Tavares (2004) na área de estudo e por Tenenbaum et al. (2006) no Rio de Janeiro.

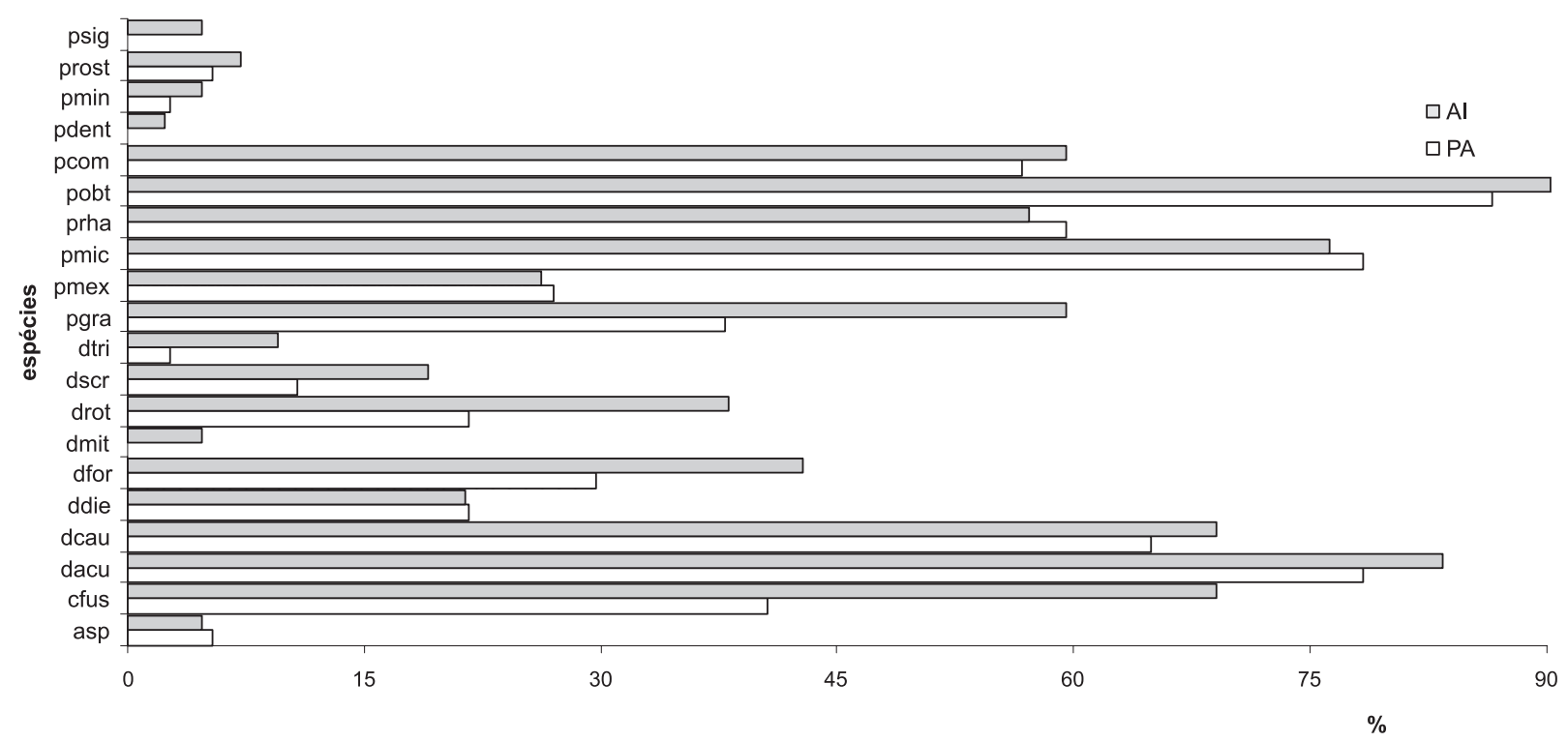

Figura 12 - Freqüência de ocorrência das espécies encontradas nos dois pontos amostrados, Al e PA nas amostras de rede entre o período de abril de 2007 à abril de 2008. Legenda: asp- A cf. tamarense, cfus- $C$. fusus, dacu- $D$. acuminata, dcau- $D$. caudata, ddie- $D$. diegensis, dfor- D. fortii, dmit- D. mitra, drot- D. rotundata, dscr- D. scrobiculata, dtri- D. tripos, pgra- P. gracile, pmex- P. mexicanum, pmic- P. micans, prha- P. rhathymum, pobt- P. obtusum, pcom- P. compressum, pmin- P. minimum, pden- P. dentatum, prost- P. rostratum, psig- P. sigmoide. 


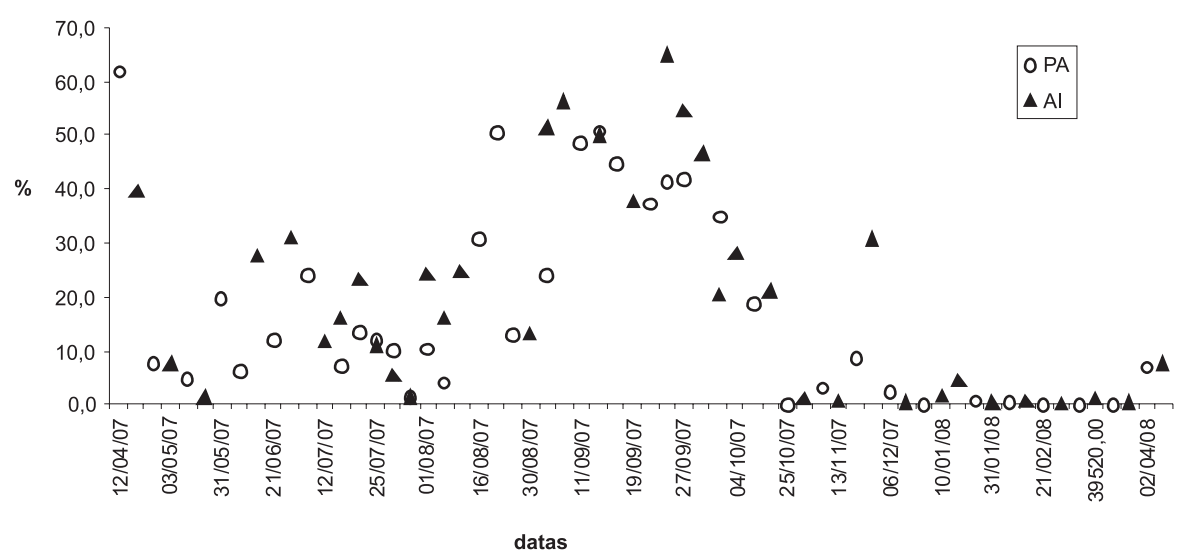

Figura 13 - Abundância relativa dos dinoflagelados tecados nocivos à presença de outras microalgas nos pontos $\mathrm{Al}$ e $\mathrm{PA}$, no período de abril de 2007 a abril de 2008.

\section{Espécies freqüentes}

D. caudata está distribuída em águas tropicais e temperadas e parece ser abundante em águas costeiras (Taylor et al., 2003). Foi registrada por Cardoso (1998) e Tavares (2004) em Santa Catarina e por Tenenbaum et al. (2004) no Rio de Janeiro. As células encontradas apresentaram 50-52 $\mu \mathrm{m}$ de comprimento e 40-42,5 $\mu \mathrm{m}$ de largura, sendo menores que as descritas na literatura, também foram observadas células pareadas (em divisão) unidas pela expansão dorsal, consideradas comuns por Faust \& Gulledge (2002).

$D$. fortii é uma espécie tóxica associada a eventos de DSP, distribuída em águas quentes e temperadas, mas também em águas subtropicais e tropicais (Faust \& Gulledge, 2002). Segundo Balech (2002) é uma espécie rara ao sul do Brasil, sendo mais freqüente ao norte. Nas amostradas analisadas, $D$. fortii esteve mais freqüente no período de final de agosto ate início de novembro de 2007.

$P$. compressum é uma espécie bastante variável, parece ser cosmopolita (Balech, 1988). No Brasil foi descrita por Cardoso $(1994,1998)$ em Santa Catarina e por Tenenbaum et al. (2006) no Rio de Janeiro. Nas amostras analisadas esteve ausente nos meses de abril a junho de 2007, ocorrendo praticamente em todas as amostras de julho a outubro de 2007 . O comprimento das células observadas no presente estudo variou e 36-42,5 $\mu \mathrm{m}$ e a largura 30-35 $\mu \mathrm{m}$.

A espécie $P$. rhathymum, é considerada por muitos autores como sendo sinônimo de $P$. mexicanum, porém características morfológicas evidentes distinguem essas duas espécies. As células de $P$. rhathymum encontradas apresentaram 45-50 $\mu \mathrm{m}$ de comprimento e 35-40 $\mu \mathrm{m}$ de largura. São muito parecidas com $P$. mexicanum, porém, as células são ovais e não há a presença de um pirenóide central.

$P$. rhathymum é uma espécie produtora de toxina hemolítica e toxina "fast-acting" (Taylor et al.,
2003). Não é considerada uma espécie de dinoflagelado que pode causar envenenamento através do consumo de moluscos contaminados, entretanto, já foi identificada como tóxica, e a mortalidade de moluscos já foi associada a outros membros do grupo (Pearce et al., 2005). No Brasil, não há registros da presença de $P$. rhathymum, sendo este o primeiro registro da espécie na costa do país.

$P$. gracile é uma espécie confundida com $P$. micans, possuindo características que se sobrepõem (Cohen-Fernandez et al., 2006), se distingue por ser relativamente estreito e pela presença de um espinho quase sempre mais alongado (Balech, 1988). Sua ocorrência já foi registrada por Tenenbaum et al. (2004) no Rio de Janeiro e por Rörig et al. (1998) e Tavares (2004) na área de estudo. No período estudado, $P$. gracile foi encontrado mais freqüentemente nas amostras de abril e maio de 2007 e a partir de novembro de 2007 até abril de 2008.

N. fusus é uma espécie variável e de distribuição muito grande. É cosmopolita (Balech, 1988), ocorrendo em águas costeiras onde pode tolerar grandes variações de salinidade (Taylor et al., 2003). Foi descrito por Cardoso (1998), Tenenbaum et al. (2006), Koening \& Lira (2005) e na área de estudo por Tavares (2004). Não é uma espécie produtora de toxinas, porém florações de $N$. fusus, podem causar morte de organismos marinhos por obstrução branquial devido à sua forma.

\section{Espécies pouco freqüentes}

A espécie Dinophysis rotundata é um dinoflagelado tóxico amplamente distribuído em águas quentes e frias (Faust \& Gulledge, 2002). É uma espécie produtora de DSP, porém, não há registros de florações (Larsen \& Moestrup, 1992). Sua ocorrência já foi descrita na área de estudo por Tavares (2004). $D$. scrobiculata é considerada segundo Balech $(1988,2002)$ praticamente idêntica a $D$. rotundata. 
Não é considerada uma espécie tóxica, porém, pela sua grande semelhança com $D$. rotundata é de grande importância sua presença neste trabalho.

$D$. diegensis é considerada uma parte do ciclo de vida de $D$. caudata, sendo sua presença associada a $D$. caudata. As espécies encontradas nas amostras apresentaram 30-35 $\mu \mathrm{m}$ de comprimento e 25-32,5 $\mu \mathrm{m}$ de largura. Na Enseada de Armação do Itapocorói, $D$. diegensis já foi escrita por Tavares et al. (2004), porém foi considerada esporádica, ocorrendo em apenas duas amostras.

$P$. mexicanum é uma espécie de dinoflagelado bentônico comumente encontrado em águas tropicais e subtropicais (Steidinger \& Tangen, 1996) e em lugares protegidos em áreas dos oceanos Pacífico e Atlântico (Faust, 1999). As células podem nadar livremente ou se unem a detritos flutuantes com um muco típico (Faust \& Gulledge, 2002). No Brasil, uma cepa de $P$. mexicanum do litoral de São Paulo, foi testada por Naves et al. (2006) comprovando sua toxicidade e sugerindo a presença de citotoxinas com mecanismo de ação semelhante ao ácido okadáico, entretanto, mais estudos são necessários para comprovação. Sua presença também foi registrada no Rio de Janeiro por Tenenbaum et al. (2004) e em Santa Catarina ainda não há registros da presença de $P$. mexicanum, sendo este o primeiro registro da espécie para o estado. As células encontradas variaram de $50-55 \mu \mathrm{m}$ de comprimento e $40-45 \mu \mathrm{m}$ de largura, sendo maiores que as descritas na literatura por Cortés-Altamirano \& Sierra - Beltrán (2003).

\section{Espécies esporádicas}

A espécie Dinophysis tripos está amplamente distribuída em águas tropicais e subtropicais e ocasionalmente é encontrada em regiões frias (Larsen \& Moestrup, 2002), mas comumente encontrada em ambientes neríticos, estuarinos e oceânicos (Steindinger \& Tangen, 1996, Faust \& Gulledge, 2002). É associada a eventos de DSP, porém não há relatos de florações (Faust \& Gulledge, 2002). Foi registrada por Tavares (2004) na área de estudo. No presente trabalho foram encontradas células de $D$. tripos pareadas, unidas pela porção dorsal. Seu tamanho variou de 57,5-60 $\mu \mathrm{m}$ de comprimento e 40-50 $\mu \mathrm{m}$ de largura, sendo menores que as encontradas na literatura.

$D$. mitra foi encontrada em três ocasiões nas amostras, no ponto $\mathrm{Al}$ em 28/06/07 e 27/02/08 e no ponto PA em 11/10/07. É uma espécie tóxica associada à DSP, amplamente distribuída em águas quentes (Faust \& Gulledge, 2002), não tendo nenhum registro de florações (Larsen \& Moestrup, 2002).

$P$. minimum é uma espécie tóxica, produtora de uma substância denominada venerupina, encontrado em águas salobras, frias, temperadas a tropicais
(Faust \& Gulledge, 2002). É responsável por contaminação de moluscos no Japão, Golfo do México e Flórida (Steindinger \& Tangen, 1996). As florações parecem ocorrer, na maioria das vezes, em água salobra (Faust et al., 1999). Na área de estudo de Armação do Itapocorói, já havia sido registrado por Rörig et al. (1998) e por Tavares (2004). No presente estudo, $P$. minimum ocorreu em apenas três amostras no mês de julho de 2007. As células encontradas variaram de 15-17,5 $\mu \mathrm{m}$ de comprimento e 14-17 $\mu \mathrm{m}$ de largura. Segundo Faust \& Gulledge (2002) P. minimum é provavelmente perdido ou ignorado freqüentemente em amostras de ambiente, devido ao seu pequeno tamanho.

$P$. rostratum é uma espécie de água quente, com registros nos oceanos Atlântico, Pacífico e Índico e no Mar Mediterrâneo (Schiller, 1933). Nas amostras analisadas, $P$. rostratum foi observado apenas nos meses de fevereiro e março de 2008. Sua presença também foi registrada por Cardoso (1998) em Santa Catarina.

$P$. dentatum é uma espécie oceânica ou nerítica, distribuída ao redor de todo o mundo (Fukuyo et al., 1990). Apresentou a menor freqüência de ocorrência, estando presente em apenas uma amostra em 25/07/07.

P. sigmoide é amplamente distribuído em águas tropicais e subtropicais ao redor do mundo. É uma espécie não-tóxica, nociva a organismos marinhos devido à depleção do oxigênio dissolvido quando em altas concentrações. Foi descrito por Tenenbaum et al. (2006) no Rio de Janeiro, não sendo ainda registrado na área de estudo. Apresentou uma freqüência de ocorrência baixa, só estando presente em três amostras.

Espécies pertencentes ao "Complexo tamarense" estão associadas a florações tóxicas de PSP em regiões de águas costeiras frias (Steindinger \& Tangen, 1996). Entretanto, têm sido registradas em águas quentes ao redor do mundo como na Austrália, Venezuela, Equador, Golfo do México e Tailândia (Balech, 2002), Portugal, EUA, Canadá, do litoral da Argentina a Patagônia (Balech, 1995).

$\mathrm{Na}$ América do Sul, a primeira floração de uma espécie pertencente a este complexo, Alexandrium tamarense, foi registrada no Uruguai em 1991 (Brazeiro et al., 1997), e no Brasil, foi registrado no Rio Grande do Sul em 1996 (Odebrecht et al., 2002). Para Santa Catarina, caso confirmado será o primeiro registro da ocorrência do dinoflagelado tecado produtor de PSP. As células encontradas no trabalho ocorreram nos dias 19 e 26 de abril, 03 de maio e 14 de junho de 2007, apresentaram uma média de $32,5 \mu \mathrm{m}$ de comprimento e $27,5 \mu \mathrm{m}$ de largura. A placa sulcal posterior "sp" e o complexo do poro apical "APC" não 
apresentaram poros auxiliares e não foi possível visualizar a presença do poro ventral na margem direita da primeira placa "1'", o que não nos permitiu confirmar sua identificação. Pode-se atribuir sua ocorrência além de outros fatores, o período sob influência das correntes predominantes do sul, podendo trazer algumas espécies deste grupo para o estado.

Resultados de abundância relativa demonstraram que na área pesquisada, os dinoflagelados tecados nocivos foram considerados abundantes quando comparados à presença de outras espécies de microalgas. Apesar da ocorrência da floração de $D$. acuminata, pôde-se observar que durante este período, a abundância relativa dos dinoflagelados tecados potencialmente tóxicos e nocivos foi elevada, pela dominância conjunta das espécies do gênero Prorocentrum.

$\mathrm{Na}$ maioria das amostras, o fitoplâncton apresentou-se dominado por microalgas de diversas espécies, sendo que no período do início do inverno até a primavera, ocorreu um aumento na abundância relativa dos dinoflagelados, também relatado por Rörig et al., (1998).

A similaridade entre as espécies nos dois pontos amostrados foi alta, devido a uma grande semelhança na morfodinâmica dos ambientes. A grande diferença na similaridade das espécies nos meses de junho (10\%) e julho (91\%), pode ser explicada pelo aumento brusco da riqueza de espécies no dia 28/06/07, que passou de 5 para 14 espécies, mantendo uma média de 9 espécies por amostragem no mês de julho. Este aumento provavelmente ocorreu por uma disponibilização de nutrientes que provocaram a proliferação de dinoflagelados, além de outras condições ambientais favoráveis. Neste período, início do inverno até a primavera, foi observado um aumento no número de dinoflagelados na área de estudo, coincidindo com as típicas florações que ocorrem nesta época em latitudes medias e altas (Rörig et al., 1998).

Este estudo mostrou um pouco da dinâmica dos dinoflagelados tecados, onde se pôde observar que é alto o risco de ocorrência de futuras florações, pela ampla diversidade de espécies deste grupo, sendo de grande importância a identificação destas espécies em áreas de maricultura.

Pode-se observar também a ocorrência de espécies ainda não descritas para a área de estudo, como P. rhathymum, P. mexicanum e Complexo tamarense.

Desta forma, conclui-se que há uma necessidade de um monitoramento constante nas áreas de maricultura do estado, sendo reforçada pela dependência econômica da população local, pelas atividades de maricultura além do turismo e da pesca.

\section{REFERÊNCIAS}

Balech, E.; Akselman, R.; Benavides, H.R. \& Negri, R.M. 1984. Suplemento a Los Dinoflagelados del Atlantico Sudoccidental. Mar del Plata: INIDEP.

Balech, E. 1988. Los Dinoflagelados del Atlántico Sudoccidental. Madrid, Publ. Espec. Oceanografia $\mathrm{n}^{\circ} 1.310 \mathrm{p}$.

Balech, E. 1995. The genus Alexandrium Halim (Dinoflagellata). Sherkin Island Marine Station, Special Publication. Cork, Ireland, 151p.

Balech, E. 2002. Dinoflagelados tecados tóxicos del Cono Sur Americano. In: Sar, E.A.; Ferrario, M.E. \& Reguera, B. (eds.). Floraciones Algales Nocivas en el Cono Sur Americano. Vigo: Instituto Español de Oceanografia. 125-144pp.

Brazeiro, A.; Méndez, S. \& Ferrari, G. 1997. The first toxic bloom of Alexandrium tamarense in Uruguai: Associated environmental factors. Atlântica: Rio Grande, 19: 19-29.

Cardoso, L.S.; Baptista, L.R. de M. \& Jebram, D.H.A. 1994. Dinoflagelados da llha do Arvoredo e da Praia de Ponta das Canas - SC, Brasil (setembro de 1991 a fevereiro de 1992). Porto Alegre: Iheringia, Ser. Bot. 45: 143-55.

Cardoso, L.S. 1998. Dinoflagelados da Ilha do Arvoredo e da Praia de Ponta das Canas, Santa Catarina, Brasil. Porto Alegre: Biociências, 6; 3-54.

Cohen-Fernandez, E.; Castillo, E.M.Del.; Ugarte, I.H. \& Pedroche, F.F. 2006. Contribuition of external morfology in solving a species complex: The case of Prorocentrum micans, Prorocentrum gracile and Prorocentrum sigmoides (Dinoflagellata) from de Mexican Pacific Coast. Phycol. Res. 54(3): 30-340.

Cortés-Altamirano, R. \& Sierra-Beltrán, A.P. 2003. Morphology and taxonomic of Prorocentrum mexicanum and Prorocentrum rhathymum (Dinophyceae). J. Phycol. 39: 221-225.

Faust, M.A.; Larsen, J. \& Moestrup, O. 1999. Potentially Toxic Phytoplancton, Genus Prorocentrum (Dinophyceae). In: Lindley, J.A. (Ed). ICES Identification Leafles for Plankton. Copenhagen. Denmark. 138-143pp.

Faust, M.A. \& Gulledge, R.A. 2002. Identifying Harmful Marine Dinoflagellates. Smithsonian Contributions from the United States Herbarium. Washington, DC. 42: 1-144pp.

Fukuyo, Y.; Takano, H.; Chihara, M. \& Matsuoka, K. 1990. Red Tide Organisms in Japan - An Illustrated Taxonomic Guide. Tokyo: Uchida Rokakuho, 430p.

Guimarães, S.C.P. \& Rörig, L.P. 2004. Efeito da salinidade no crescimento dos dinoflagelados Prorocentrum micans Ehrenberg e Prorocentrum cf. obtusum Ostenfeld isolados da costa Catarinense - Brasil. Estud. Biol., 26(54): 29-36. 
Harsen, G.; Turquet, J.; Quod, J.P.; Ten-Hage, L.; Lugomela, C.; Kyewalyanga, M.; Hulburns, M; Wawiye, P.; Ogongo, B.; Tunje, S. \& Rakotoarinjanahary, H. 2001. Potentially Harmful Microalgae of the westen Indian Ocean - a guide based on a preliminary survey. UNESCO. 105p.

Koening, M.L. \& Lira, C.G.de. 2005. O gênero Ceratium Schrank (Dinophyta) na plataforma continental e águas oceânicas do Estado de Pernambuco, Brasil. Acta Bot. Bras. 19(2): 391-397.

Larsen, M.D. \& Moestrup, O. 1992. Potentially Toxic Phytoplancton, Genus Dinophysis (Dinophyceae). In: Lindley, J.A. (Ed). ICES Identification Leafles for Plankton. Copenhagen. Denmark:

Larsen, J. \& Ngueyen, N.L. 2004. Potentially toxic microalgae of Vietnamese waters. In: Opera Botanica 140. Compenhagen. Denmark: Data Center.

Lobo, E. \& Leighton, G. 1986. Estructuras de las fitocenosis planctônicas de los sistemas de desembocaduras de rios y esteros de la zona central do Chile. Rev. Biol. Mar. 22: 143-170.

Lopez-Rodas, V.; Manerio, E.; Martinez, J.; Navarro, M. \& Costas, E. 2006. Harmful algal blooms, red tides and human health: Diarrhetic shellfish poisoning and colorectal cancer. An. R. Acad. Nac. Farm., 72: 391-408pp.

Mafra Jr., L.L.; Fernandes, L.F. \& Proença, L.A.O. 2006. Harmful algae and toxinas in Paranaguá Bay, Brazil: bases for monitorig. Braz. J. Oceanog. 54: 107-121.

Manerio, E.; Rodas, V.L.; Costas, E. \& Hernandez, J.M. 2008. Shellfish consumption: A major risk factor for colorectal cancer. Med. Hypotheses 70: 409-412.

Marenzi, A.W.C. \& Branco, J.O. 2006. O cultivo do mexilhão Perna perna no município de Penha, SC. In: Branco, J.O. \& Marenzi, A.W.C. (Org.). Bases ecológicas para um desenvolvimento sustentável: estudos de caso em Penha, SC. 291. Editora da UNIVALI, Itajaí, SC. 227-244pp.

Naves J.L.; Prado, M.P.; Rangel, M.; Sanctis, B.; Machado-SantellI, GM. \& Freitas, J.C. 2006. Cytotoxicity in the marine dinoflagellate Prorocentrum mexicanum from Brazil. Comp. Biochem. Physiol. C Toxicol. Pharmacol. 143(1): 73-77.

Odebrecht, C.; Azevedo, S.M.F.O.; Garcia, V.M.T.; Huszar, V.L.M.; Magalhães, V.F.; Menezes, M.; Proença, L.A.O.; Rörig, L.R.; Tenenbaum, D.R.; Vilac, M.C. \& Yunes, J.S. 2002. Floraciones de Microalgas Nocivas en Brasil: Estado del Arte y Proyetos en Curso. In: Floraciones Algales Nocivas em El Cono Sur Americano. Instituto Español de Oceanografia, Vigo. 217-234pp.
Oliveira Neto, F.M. 2007. Síntese informativa da produção de moluscos (mexilhões, ostras e vieiras) no estado de Santa Catarina em 2006. Florianópolis, 2007. Disponível em: < http://www. epagri.rct-sc.br/ >. Acesso em: 09 de ago. 2007.

Omori, M. \& Ikeda, T. 1984. Methods on marine zooplankton ecology. Jonh wiley and Sons Publ. NewYork. 332 p.

Panorama da Aquicultura, 2001. Panorama da malacocultura brasileira. 11(64): 25-31.

Pearce,I.; Handlinger, J.H. \& Hallegraeff, G.M. 2005. Histopathology in Pacifc oyster (Crossostrea gigas) spat caused by the dinoflagellate Prorocentrum rhathymum. Harmful Algae 4: 61-74.

Proença, L.A.O.; Schimitt, F.; Costa, T. \& Rörig, L. 1998. Just a diarrhea? Evidences of diarrhetic shellfish poisoning in Santa Catarina - Brazil: Ciênc. Cult. 51: 16-21.

Proença, L.A.O.; Schimitt, F.; Guimarães, S.P. \& Rörig, L. 1999. Análise de toxinas diarréicas em duas espécies de Prorocentrum (Diophyceae) em área de cultivo de moluscos. Notas tec. FACIMAR. 3: 41-45.

Proença, L.A.O.; Schramm, M.A.; Tamanaha, M.S. \& Alves, T.P. 2007. Diarrhoetic Shellfish Poisoning (DSP) outbreak in Subtropical Southwest Atlantic. Harmful Algae News. 33: 19-20.

Rörig, L.R.; Guimarães, S.C.P.; Lugli, D.O.; Proença, L.A.O.; Manzoni, G.C. \& Marenzi, A.C. 1998. Monitorização de microalgas planctônicas potencialmente tóxicas na área de maricultura da Enseada de Armação do Itapocorói - Penha, SC. Notas Tec. FACIMAR 2: 71-79.

Schettini, C. A. F.; Carvalho, J. L. \& Truccolo, E. C. 1999. Aspectos hidrodinâmicos da Enseada de Armação do Itapocoroy, SC. Notas Tec. FACIMAR, 3: 99-109.

Schiller, J. Dinoflagellatae 1933. (Peridineae) In: Monographisher behandlung. Akademsche Verlagsesell Schaft. M.B.H.

Schimitt, F. \& Poença, L.A.O. 1999. Ocorrência de dinoflagelados do gênero Dinophysis (Enrenberg, 1839) na Enseada de Cabeçudas (verão e outono de 1999). Notas tec. FACIMAR 4: 49-59p.

Steindinger, K.A. \& Tangen K.. 1997. Dinoflagellates. In: Tomas, C.R. (Ed.) Identifying Marine Phytoplancton. California: Academic Press. 387-584 p.

Tavares, J.F.R. 2004. Microalgas potencialmente nocivas na área de maricultura de Armação do Itapocorói SC, Brasil. Trabalho de conclusão de curso (Graduação em Oceanologia). Fundação Universidade do Rio Grande. Rio Grande, 136p.

Taylor, F.J.R.; Fukuyo, Y.; Larsen, J. \& Hallegraeff, G.M. 2003. Taxonomy of harmful dinoflagellates. In: Manual On Harmful Marine Microalgae, United 
Nations Educational, Scientific and Cultural Organization; Paris. 389-432pp.

Tenenbaum, D.R.; Villac, M.C.; Viana, S.C.; Matos, H.; Hatherly, M.; Lima, I.V. \& Menezes, M. 2004. Phytoplankton Atlas of Sepetiba Bay, Rio de Janeiro, Brasil. Globallast Monograph, $n^{\circ}$ 16. IMO London.

Tenenbaum, D. R.; Menezes, M.; Viana, S. de Castro ; Mendes, M. C. de Queiroz ; Hatherly, M.; Eduardo, J. 2006. Os Dinoflagelados.. In: Denise Rivera
Tenenbaum. (Org.). Dinoflagelados e Tintinídeos da costa central na Zona Econômica Exclusiva brasileira. 1 ed. Rio de Janeiro: Museu NacionalUniversidade Federal do Rio de Janeiro, 37-163 pp.

Submetido: Junho/2011 Revisado: Outubro/2011 Aceito: Outubro/2011 